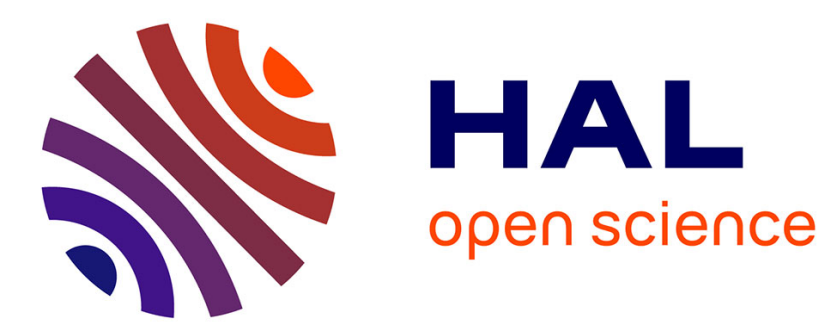

\title{
InGaN/GaN core/shell nanowires for visible to ultraviolet range photodetection
}

Hezhi H Zhang, Agnès Messanvi, Christophe Durand, Joël Eymery, Pierre Lavenus, Andrey Babichev, François H Julien, Maria Tchernycheva

\section{- To cite this version:}

Hezhi H Zhang, Agnès Messanvi, Christophe Durand, Joël Eymery, Pierre Lavenus, et al.. InGaN/GaN core/shell nanowires for visible to ultraviolet range photodetection. physica status solidi (a), 2016, 213 (4), pp.936-940. 10.1002/pssa.201532573 . hal-01390994

\section{HAL Id: hal-01390994 \\ https://hal.science/hal-01390994}

Submitted on 4 Nov 2016

HAL is a multi-disciplinary open access archive for the deposit and dissemination of scientific research documents, whether they are published or not. The documents may come from teaching and research institutions in France or abroad, or from public or private research centers.
L'archive ouverte pluridisciplinaire HAL, est destinée au dépôt et à la diffusion de documents scientifiques de niveau recherche, publiés ou non, émanant des établissements d'enseignement et de recherche français ou étrangers, des laboratoires publics ou privés. 


\title{
InGaN/GaN core/shell nanowires for visible to ultraviolet range photodetection
}

\author{
Hezhi Zhang ${ }^{*}, 1$, Agnès Messanyi ${ }^{1,2,3}$, Christophe Durand ${ }^{2,3}$, Joël Eymery ${ }^{2,3}$, Pierre Lavenus ${ }^{1}$, Andrey \\ Babichev $^{4,5}$, François H. Julien ${ }^{1}$, Maria Tchernycheva \\ ${ }^{1}$ Institut d'Electronique Fondamentale, UMR CNRS 8622, University Paris Sud 11, 91405 Orsay, France \\ ${ }^{2}$ Univ. Grenoble Alpes, 38000 Grenoble, France \\ ${ }^{3}$ CEA, INAC-SP2M, "Nanophysique et semiconducteurs" group, 38000 Grenoble, France \\ ${ }^{4}$ ITMO University, 197101 Saint-Petersburg, Russia \\ ${ }^{5}$ Ioffe Institute, Polytechnicheskaya 26, 194021 St. Petersburg, Russia
}

Keywords core/shell nanowire, InGaN/GaN, photodetector, MOVPE

*Corresponding author: e-mail hezhi.zhang@u-psud.fr

\begin{abstract}
We report on the fabrication and characterization of single nitride nanowire visible-to-ultraviolet $\mathrm{p}$-n photodetectors. Nitride nanowires containing $30 \mathrm{InGaN} / \mathrm{GaN}$ radial quantum wells with $18 \%$ indium fraction were grown by catalyst-free metal-organic vapour phase epitaxy. Single nanowires were contacted using optical lithography. As expected for a radial p-n junction, the current-voltage (I-
\end{abstract}

\begin{abstract}
V) curves of single wire detectors show a rectifying behavior in the dark and a photocurrent under illumination. The detectors present a response in the visible to UV spectral range starting from $2.8 \mathrm{eV}$. The peak responsivity is $0.17 \mathrm{~A} / \mathrm{W}$ at $3.36 \mathrm{eV}$. The on-off switching time under square light pulses is found to be below $0.1 \mathrm{sec}$.
\end{abstract}

1 Introduction InGaN/GaN two-dimensional quantum well (QW) superlattices have been intensively studied as materials for visible and ultraviolet light emission and detection $^{1-3}$. They are also investigated in view of photovoltaic conversion ${ }^{4,5}$. However, the performance of InGaN devices is negatively affected by the presence of a large number of threading dislocations ${ }^{6,7}$ and by a strong internal polarization field in c-oriented thin films ${ }^{8,9}$. Nanowires (NWs) can solve these two major problems. The dislocation density is drastically reduced due to the free lateral surface, which allows efficient relaxation of the misfit strain. For the core/shell QWs grown on the non-polar mplane facets the potential profile is not distorted by the internal field leading to a good overlap between the electron and hole wavefunctions. In addition, the NW design presents promising advantages for photodetectors ${ }^{10,11}$ because of independent optimization of light absorption and carrier collection, efficient light trapping and thus a possibility to reduce the active material volume without loosing efficiency. Previously, NW-based core/shell n-i-n InGaN/GaN detectors have been investigated ${ }^{12}$. Thanks to the high photoconductive gain, these devices exhibit a very high sensi- tivity (in the $10^{4} \mathrm{~A} / \mathrm{W}$ range), however their operation speed is low (return to equilibrium time constants are in the range of tens to hundreds of seconds). Core-shell $\mathrm{p}-\mathrm{n}$ GaN single nanowires containing a thick $(50 \mathrm{~nm}) \mathrm{InGaN}$ layer have been also used as photodetectors in a photonic platform ${ }^{10}$. The replacement of the photoconductive $n-i-n$ device by a $p-n$ junction photodiode allowed to improve the operation speed (reported characteristic times were shorter than $0.1 \mathrm{sec}$ ). However, due to the strain accumulation, the structural quality of the InGaN radial layers is quite low leading to important non-radiative losses. The material quality can be improved by replacing a thick InGaN layer with a multi-quantum well heterostructure. Up to date, there have been no reports on core/shell p-n InGaN/GaN NW photodetectors using QWs for the light absorption.

In this work, we report for the first time the fabrication of a p-n junction NW photodiode containing radial InGaN/GaN QWs and we investigate the optoelectronic properties of these devices for their application in visible to UV range photodetection. The NWs were grown by catalyst-free Metal-organic vapour phase epitaxy (MOVPE) on 
sapphire substrates. The photoresponse was studied on a model case of a single NW contacted by means of optical lithography. The NW detectors present a response in the visible to UV spectral range starting from $2.8 \mathrm{eV}$. The peak responsivity is $0.17 \mathrm{~A} / \mathrm{W}$ at $3.36 \mathrm{eV}$. This value corresponds to a more than two times enhancement with respect to the previous report for core/shell $\mathrm{p}-\mathrm{n}$ photodiodes based on a thick InGaN radial layer ${ }^{10}$. The on-off switching time under square light pulses is found to be below $0.1 \mathrm{sec}$, which is much shorter than the typical response time of photoconductive detectors suffering from a slow current decay.

2 Experimental The N-polar GaN wires were grown by MOVPE on c-sapphire substrates using in-situ $\mathrm{SiNx}$ thin film pre-deposition ${ }^{13,14}$. The bottom wire part $(\sim 10$ $\mu \mathrm{m}$ long) was heavily $\mathrm{n}^{+}$-doped. Then a non-intentionally doped GaN segment ( $10-15 \mu \mathrm{m}$ long, residual $\mathrm{n}$-doping $\sim 10^{18} \mathrm{~cm}^{-3}$ ) was deposited. The upper part of the wires was coated with $30 \mathrm{InGaN} / \mathrm{GaN}$ radial QWs $(6 \mathrm{~nm} / 24 \mathrm{~nm})$ along nonpolar m-plane facets and then overgrown with a p-doped GaN shell layer as reported in Refs ${ }^{13}, 15$. The targeted indium composition in the QWs is $\sim 18 \%$. The NW morphology is illustrated in figure 1 . The average wire length is $20-25 \mu \mathrm{m}$ and the diameter is $1-2 \mu \mathrm{m}$.

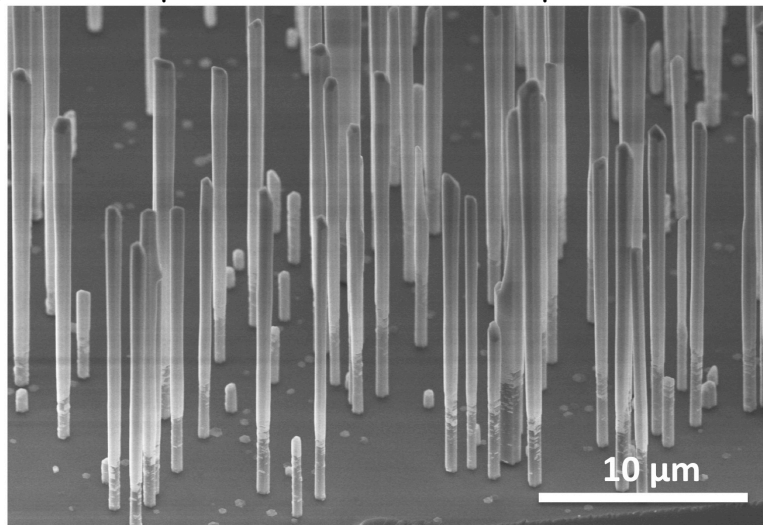

Figure (1) $45^{\circ}$ tilted SEM image of self-assembled InGaN/GaN nanowires.

To fabricate single wire photodetectors, the NWs were detached from their native substrate by ultra-sonic cutting and dispersed on a $\mathrm{SiO}_{2} / \mathrm{Si}$ substrate. The NWs were then encapsulated in a hydrogen silsesquioxane polymer (HSQ) transformed into $\mathrm{SiOx}$ after thermal baking for 1 hour at $400{ }^{\circ} \mathrm{C}$. The excess of HSQ was etched in diluted $\mathrm{HF}$ solution ( $\mathrm{HF}: \mathrm{H}_{2} \mathrm{O}=1: 200$ volume) to expose the lateral NW facet for contacting. The first contact was defined on the $\mathrm{p}-\mathrm{GaN}$ shell by optical lithography. The $\mathrm{Ni}$ / Au (10 nm / $150 \mathrm{~nm})$ was deposited followed by an annealing at $500{ }^{\circ} \mathrm{C}$ in ambient atmosphere for $5 \mathrm{~min}$ to favor the formation of ohmic contact on p-type GaN. The second electrode to n-type $\mathrm{GaN}$ core was also defined by optical lithography and Ti/Al/Ti/Au (10/20/10/200 nm) metallization. A scanning electron microscopy (SEM) image of a typical device is shown in figure 2 (a) and a schematic picture illustrating the device structure is presented in figure 2 (b).
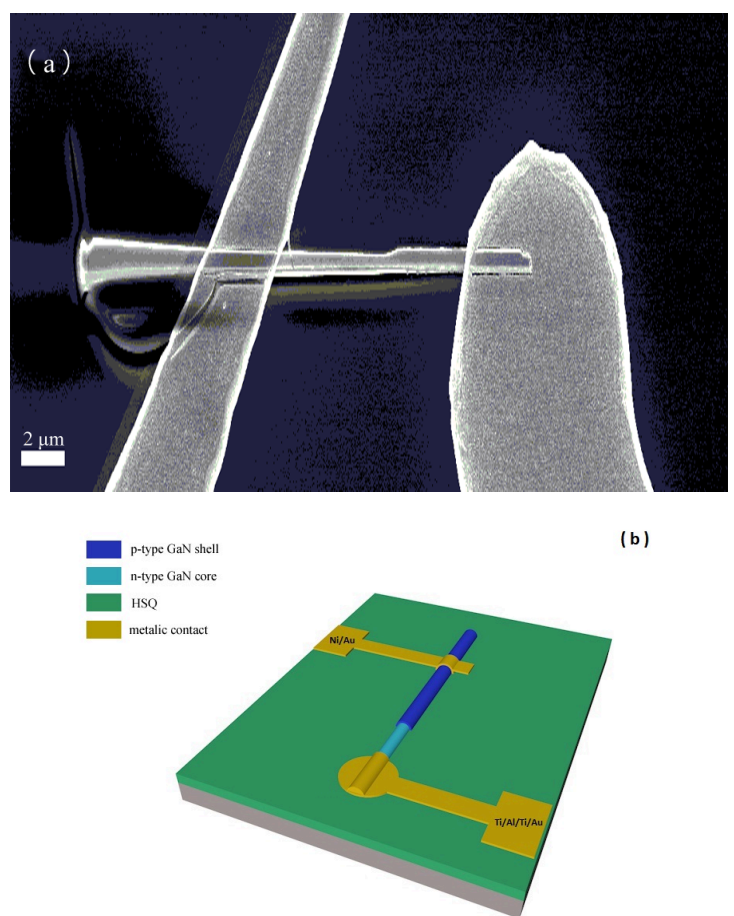

(b)

Figure 2 (a) SEM picture of the processed single nanowire visible-UV photodetector and (b) the schematic picture of a single nanowire photodetector

\section{Electrical and optical characterization 3.1 Current-voltage characterization}

Figure 3 (a) presents the I-V curve (in logarithmic scale) of a single NW photodetector in the dark and under illumination. In the dark, the I-V curve shows a typical rectifying behavior without significant current leakage (dark current is below $10^{-11} \mathrm{~A}$ at $-1 \mathrm{~V}$ bias). The non-zero dark current at zero bias (below $1 \mathrm{pA}$ ) and the current accidents in the 0.75 to $0 \mathrm{~V}$ range are due to the measurement uncertainty of our electrical detection system. Under illumination with $\lambda=370 \mathrm{~nm}$, the photodetector exhibits a strong photocurrent. The induced current under zero bias reaches $\sim 200 \mathrm{pA}$. As expected for a $\mathrm{p}-\mathrm{n}$ junction photodiode, the current signal does not significantly change with the reverse bias (for biases from 0 to $-1.5 \mathrm{~V}$ the current remains between 200 and $240 \mathrm{pA}$ ). Under forward bias, the current under illumination first slightly decreases and then increases following the dark current behavior of a $\mathrm{p}-\mathrm{n}$ junction under forward bias. 
The I-V curve for higher forward current levels (up to $50 \mathrm{nA}$ ) is shown in figure 3 (b) (in linear scale) under illumination and in the dark. It is seen that for forward bias above $1.5 \mathrm{~V}$ the slope of the I-V curve under illumination is slightly steeper that the slope of the dark I-V curve. This indicates the variation of the access resistance under illumination (possibly, the resistance of the $\mathrm{p}-\mathrm{GaN}$ shell). It should be noted that NW photodetectors due to the surface Fermi level pinning are often characterized by strong photoconductive effects leading to an elevated photocurrent gain $^{16}$. NW photoconductors can even present persistent photoconductivity. However, as seen in figure 3 (b), in the presently-investigated case of a radial p-n junction these negative effects are minimized thanks to the core-shell geometry. Indeed, the dark and illuminated I-V curves under the forward bias are almost identical, whereas a strong current increase is expected for a NW photoconductor ${ }^{16}$.
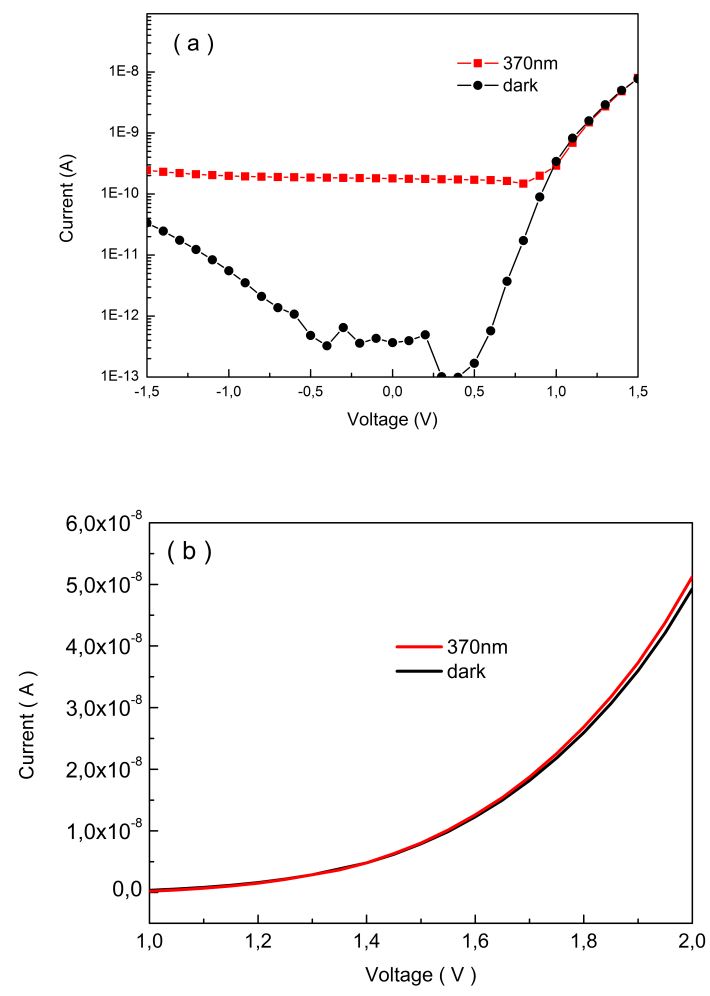

Figure 3 (a) I-V curve of a single nanowire photodetector in the dark and under illumination. (b) I-V curve for larger forward biases showing only a weak current modification under illumination.

\subsection{Time dependent photocurrent transient meas- urement}

In order to investigate the operation speed of the photodetector, the time dependent photocurrent transient measurements were performed under zero bias. The NW detector was illuminated with square light pulses with different wavelengths using a wavelength tunable light source (Xe lamp coupled with a monochromator). The photocurrent signal has been normalized with respect to the incident light intensity at different wavelengths. As shown in figure 4 (a), the photodetector presents a response to wavelengths starting from $430 \mathrm{~nm}$ (photocurrent $=4.7 \mathrm{pA}$ ) corresponding to the absorption in the $\mathrm{InGaN} / \mathrm{GaN}$ radial QWs. The response increases for shorter wavelength. The rise and the decay switching times for all illumination wavelengths are below the time resolution of the measurement system $(\sim 0.1$ $\mathrm{sec})$. We note that no persistent photoconductivity effects that would lead to slow signal transients were observed. To characterize the switching time with a better precision, the illumination was mechanically chopped at different frequencies and the detector signal was recorded. The $-3 \mathrm{~dB}$ cutoff frequency under zero bias and $\lambda=370 \mathrm{~nm}$ is found to be $30 \mathrm{~Hz}$.

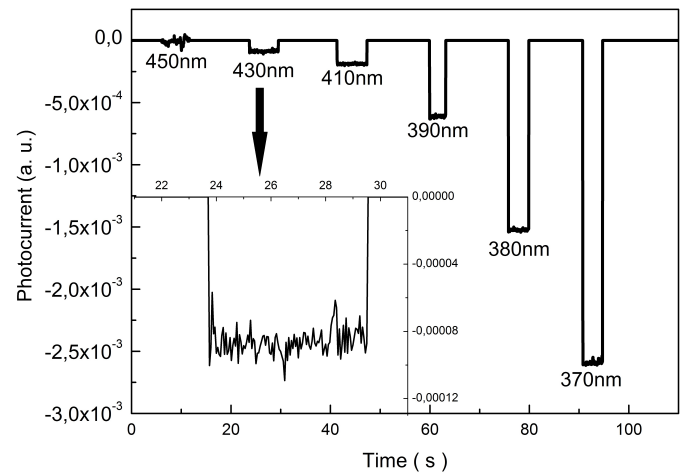

Figure 4 1 1me dependent pnotocurrent tor on/ott 11lumınation cycles at different wavelength from $450 \mathrm{~nm}$ to $370 \mathrm{~nm}$. Inset shows a close-up on one illumination cycle with $\lambda=430 \mathrm{~nm}$ corresponding to the QW absorption.

\subsection{Photocurrent spectroscopy}

To better analyze the spectral dependence of the detector reponse, photocurrent spectra of a single NW device were measured at room temperature using a wavelength tunable Xenon lamp light coupled with a Jobin Yvon Triax 180 spectrometer. The photocurrent spectrum under zero bias of a typical device in the visible-to-UV spectral range is reported in figure 5 (in a logarithmic scale). The calibration of the light source output intensity was done using a commercial calibrated photodiode sensor and the photocurrent spectrum was normalized accordingly. The detector responsivity is defined as :

$$
R=\frac{I_{p h}}{P_{\text {opt }}}
$$

where $I_{p h}$ is the photocurrent and $P_{\text {opt }}$ is the incident optical power. As seen in figure 5, the photoresponse appears below the GaN bandgap at around $2.8 \mathrm{eV}(442 \mathrm{~nm})$. This response is attributed to the absorption in the QWs and the 
subsequent extraction of the electron-hole pairs from the quantum wells by the electric field of the radial $p-n$ junction. However, the responsivity in the visible range is rather weak $(\sim 0.015 \mathrm{~A} / \mathrm{W}$ at $\lambda=442 \mathrm{~nm})$. Between $2.8 \mathrm{eV}$ and $3.36 \mathrm{eV}(370 \mathrm{~nm})$, the photocurrent rapidly increases and reaches its maximum of $0.17 \mathrm{~A} / \mathrm{W}$ at $3.36 \mathrm{eV}(\sim 370 \mathrm{~nm})$. This increase can be understood as a consequence of the inhomogeneous In compositions in the $\mathrm{QWs}^{17}$. The regions with a lower In content contribute to the photocurrent at shorter wavelengths. In addition, the carrier extraction from the shallow In poor QW parts is more efficient than from the deep In-rich QW parts. The signal remains almost constant until $3.54 \mathrm{eV}(350 \mathrm{~nm})$ and then decreases by $35-$ $40 \%$ toward the UV range $(300 \mathrm{~nm})$. This decrease for wavelengths shorter than the GaN bandgap is due to the loss of photoexcited carriers absorbed in the $\mathrm{p}-\mathrm{GaN}$ shell region. Indeed, these carriers can recombine on surface states before diffusing to the $\mathrm{p}-\mathrm{n}$ junction field region and thus they do not contribute to the photocurrent.

With respect to the previously reported NW core/shell $\mathrm{p}-\mathrm{n}$ photodiodes based on a thick radial InGaN layer ${ }^{10}$, the peak responsivity of the multi-QW photodiode is increased from $0.075 \mathrm{~A} / \mathrm{W}$ to $0.17 \mathrm{~A} / \mathrm{W}$. We attribute this enhancement to the improvement of the structural properties of the multi-QW nanowires. Indeed, thick InGaN layers exhibit plastic relaxation due to the lattice mismatch with the GaN core. Therefore, thick InGaN layers are expected to have a large number of non-radiative defects leading to carrier losses. The multi-QW design allows to improve the structural quality leading to a higher responsivity.

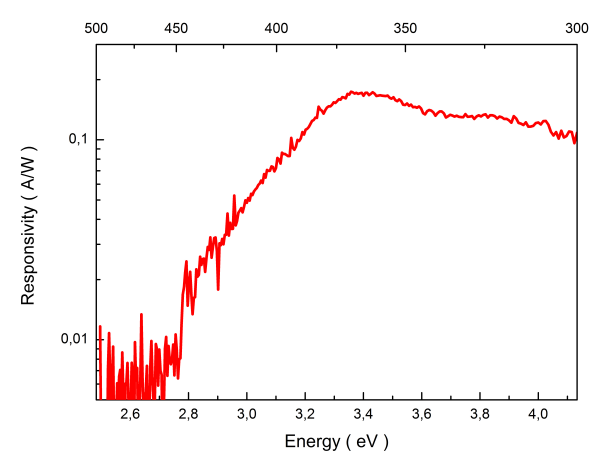

Figure 5 Room temperature photocurrent spectrum of a single nanowire photodetector.

\section{Conclusion}

In summary, we fabricated and characterized single NW photodetectors operating in the visible-to-UV spectral range. Self-assembled NWs containing $30 \mathrm{InGaN} / \mathrm{GaN}$ core/shell QWs ( $6 \mathrm{~nm} / 24 \mathrm{~nm}$ ) in the middle of a radial p$\mathrm{n}$ junction were synthesized by MOVPE. The electrical properties of the photodetector as well as the time dependent current transits and the photocurrent spectra were analyzed. Contrary to the NW photoconductors, the detector presents no persistent photoconductivity and the response time is below $0.1 \mathrm{sec}$, which is limited by experimental setup timporal resolution. The detector response extends from $2.8 \mathrm{eV}(442 \mathrm{~nm})$ to the deep UV range. The peak responsivity is $0.17 \mathrm{~A} / \mathrm{W}$ at $3.36 \mathrm{eV}(370 \mathrm{~nm})$.

Acknowledgements This work has been partially financially supported by Laboratory of Excellency 'GaNeX' (ANR11- LABX-2014) and 'NanoSaclay' (ANR-10-LABX-0035), by ANR-14-CE26-0020-01 project 'PLATOFIL', by FP7 Marie Curie projects 'Funprob' and by EU ERC project "NanoHarvest" (grant no. 639052). A. Babichev acknowledges the support of RFBR (Project number 14-02-31485, 15-02-08282), Russian Science Foundation (Project number 15-12-00027) for photoconductivity measurements support, and of the Scholarship of the President of the Russian Federation (Grant number SP4716.2015.1). The device processing has been performed at CTU-IEF-Minerve technological platform, member of the Renatech RTB network.

\section{References:}

[1] S. J. Chang, M. L. Lee, J. K. Sheu, W. C. Lai, Y. K. Su, C. S. Chang, C. J. Kao, G. C. Chi, and J. M. Tsai, IEEE Electr Device L 24, 212 (2003).

[2] J. Pereiro, C. Rivera, A. Navarro, E. Munoz, R. Czernecki, S. Grzanka, and M. Leszczynski, IEEE J Quantum Elect 45, 617 (2009).

[3] C. Rivera, J. L. Pau, J. Pereiro, and E. Muñoz, Superlattice Microst 36, 849 (2004).

[4] M. Anani, C. Mathieu, M. Khadraoui, C. Zouaoui, S. Lebid, and Y. Amar, Microelectronic J 40, 427 (2009).

[5] A. G. Bhuiyan, K. Sugita, A. Hashimoto, and A. Yamamoto, IEEE Journal of Photovoltaics 2, 276 (2012).

[6] C. J. Humphreys, Mrs Bull 33, 459 (2008).

[7] S. C. Jain, M. Willander, J. Narayan, and R. V. Overstraeten, J Appl Phys 87, 965 (2000).

[8] S. F. Chichibu, T. Sota, K. Wada, O. Brandt, K. H. Ploog, S. P. Denbaars, and S. Nakamura, phys. stat. sol. (a) 183, 91 (2001).

[9] L. H. Peng and C. W. Chuang and L. H. Lou, Appl Phys Lett 74, 795 (1999).

[10] M. Tchernycheva, A. Messanvi, A. de Luna Bugallo, G. Jacopin, P. Lavenus, L. Rigutti, H. Zhang, Y. Halioua, F. H. Julien, J. Eymery and C. Durand, Nano Lett 14, 3515 (2014).

[11] L. Rigutti, F. Fortuna, M. Tchernycheva, A. De Luna Bugallo, G. Jacopin, F. H. Julien, F. Furtmayr, M. Stutzmannand M. Eickhoff, physica status solidi (a) 207, 1323 (2010).

[12] A. De Luna Bugallo, L. Rigutti, G. Jacopin, F. H. Julien, C. Durand, X. J. Chen, D. Salomon, J. Eymery, and M. Tchernycheva, Appl Phys Lett 98, 233107 (2011).

[13] R. Koester, J. S. Hwang, D. Salomon, X. J. Chen, C. Bougerol, J. P. Barnes, D. L. S. Dang, L. Rigutti, A. De Luna Bugallo, G. Jacopin, M. Tchernycheva, C. Durand and J. Eymery, Nano Lett 11, 
4839 (2011).

[14] R. Koester, J. S. Hwang, C. Durand, D. S. Dang, and J. Eymery, Nanotechnology 21, 15602 (2010).

[15] P. Lavenus, A. Messanvi, L. Rigutti, A. De Luna Bugallo, H. Zhang, F. Bayle, F. H. Julien, J. Eymery, C. Durand, and M. Tchernycheva, Nanotechnology 25, 255201 (2014).

[16] G. Jacopin, A. De Luna Bugallo, L. Rigutti, P. Lavenus, F. H. Julien, Y. Lin, L. Tu, and M. Tchernycheva, Appl Phys Lett 104, 23116 (2014).

[17] L. Rugutti, I. Blum, D. Shinde, D. HernańdezMaldonado, W. Lefebvre, J. Houard, F. Vurpillot, A. Vella, M. Tchernycheva, C. Durand, J. Eymery, B. Deconihou, Nano Letters 14, 107-114 (2014). 